\title{
RILIEVO GEOFISICO DELL'ALTIPIANO DI ASIAGO
}

\author{
Carlo Morelli
}

1. Premessa. -- L'Istituto Nazionale di Geofisica ha in programma la creazione di un Osservatorio magnetico di $1^{\circ}$ ordine anche nell'Italia sett., che con gli altri in corso di costruzione nelle Marche, in Sicilia ed in Sardegna completi i caposaldi magnetici della nuova rete geofisica italiana.

E hen nota però la difficoltà di trovare, specie nell'Italia sett., una località che risulti idonea ad una tale costruzione.

Per suggerimento del chiar.mo prof. Giorgio Dal Piaz, esperto conoscitore della Geologia della regione, la scelta cadde sull'Altipiano di Asiago, per essere esso costituito da uno strato di calcare di notevole spessore, e per essere praticamente esente dai disturbi dovuti alle ferrovie e linee elettriche.

Senonché, come è dimostrato chiaramente dalle carte geologiche, esso ì quasi un'isola magneticamente non perturbata, circondata da manifestazioni vulcaniche: a Nord dalle enormi manifestazioni ignee intrusive (granito) del massiccio di Cima d'Asta, a Sud dalle estreme propaggini settentrionali dei Berici. Rimaneva quindi da accertare se tali manifestazioni vulcaniche non continuassero anche solto il calcare, in maniera da poter arrecare sensibili disturbi in superficie; o se addirittura nel calcare stesso non esistessero intrusioni verso la superficie.

Venne perciò deciso di effettuare un rilievo geofisico nella zona in discussione, con stazioni piì dense nella parte centrale dell'Altipiano, dove interessava anche il comportamento geofisico locale per l'eventuale scelta del posto per l'Osservatorio; e con carattere vieppiù regrionale verso $i$ margini dell'altipiano e nelle zone circostanti.

Il rilievo consta di una parte magnetica e di una parte gravimetrica, eseguite contemporaneamente dal $1^{\circ}$ al 20 agosto 1952 , con $\mathrm{i}$ criteri ed i risultati più sotto esposti.

2. Rilievo magnetico. - Le misure sono state effeltuate con variometri magnetici di Schmidt, e sono state misurate solo differenze nella componente verticale $Z$. Sono stati adoperati due variometri, la cui 
costante di scala $\varepsilon_{2}$ è stata determinata prima della campagna e controllata alla fine della stessa:

$$
\begin{aligned}
& \text { Askania n. } 509983: \varepsilon_{\mathrm{z}}=9.65 \mathrm{\gamma} / \mathrm{div} . \\
& \text { Ruska n. } 2988: \\
& \quad=9.45 \mathrm{n} / \mathrm{\prime} .
\end{aligned}
$$

Ogni strumento era corredato da due treppiedi, che venivano messi in ciascuna stazione a circa $20 \div 30 \mathrm{~m}$ uno dall'altro, allo scopo di controllare la normalità della stazione stessa.

Come hase di partenza si è assunto il picchetlo nel parco dell'Osservatorio Astrofisico di Asiago, circa $150 \mathrm{~m}$ a SE della cupola. Non disponendo ancora in Italia di una base assoluta per la $Z$, tale punto è stato collegato alla fine di griugno 1952 con l'Osservatorio magnetico di Fürstenfeldbruck, in Baviera, mediante due variometri magnetici Askania tarati con la più grande cura, e dividendo l'intervallo in tre tratte, sia per la sua ampiezza che per gli impedimenti dei confini.

La differenza misurata ̀̀ stata:

$$
\Delta Z=-1329 \quad \gamma
$$

per cui, essendo stato di valore di Fürstenfeldbruck nell'agosto 1952 $Z=0.41833$, la base di partenza ad Asiago assume il valore $Z=0.40504$.

Per la variazione diurna, si sono utilizzati i magnetogrammi della stazione registratrice con variometri Ruska, allestita provisoriamente in una cantina dell'Osservatorio Astrofisico di Asiago fin dal 23 febbraio 1952, con le seguenti sensibilità:

$$
\begin{array}{lllr}
\text { Variometro } & Z_{1} & : & 1.75 \gamma / \mathrm{mm} \\
& Z_{0} & : & 5,74 \gamma / \mathrm{mm} \\
\text { Variometro } & H_{1} & : & 5,52 \gamma / \mathrm{mm} \\
& H_{0} & : & 17.95 \gamma / \mathrm{mm} \\
\text { Variometro } & D_{1} & : & 52.47 " / \mathrm{mm} \\
& D_{0} & : & 154.49 " / \mathrm{mm}
\end{array}
$$

Sono state seguite le consuete $\left(^{1}\right)$ regole ed avvertenze per l'esecuzione delle misure, distribuendo le stazioni in circuiti chiusi. Dagli 


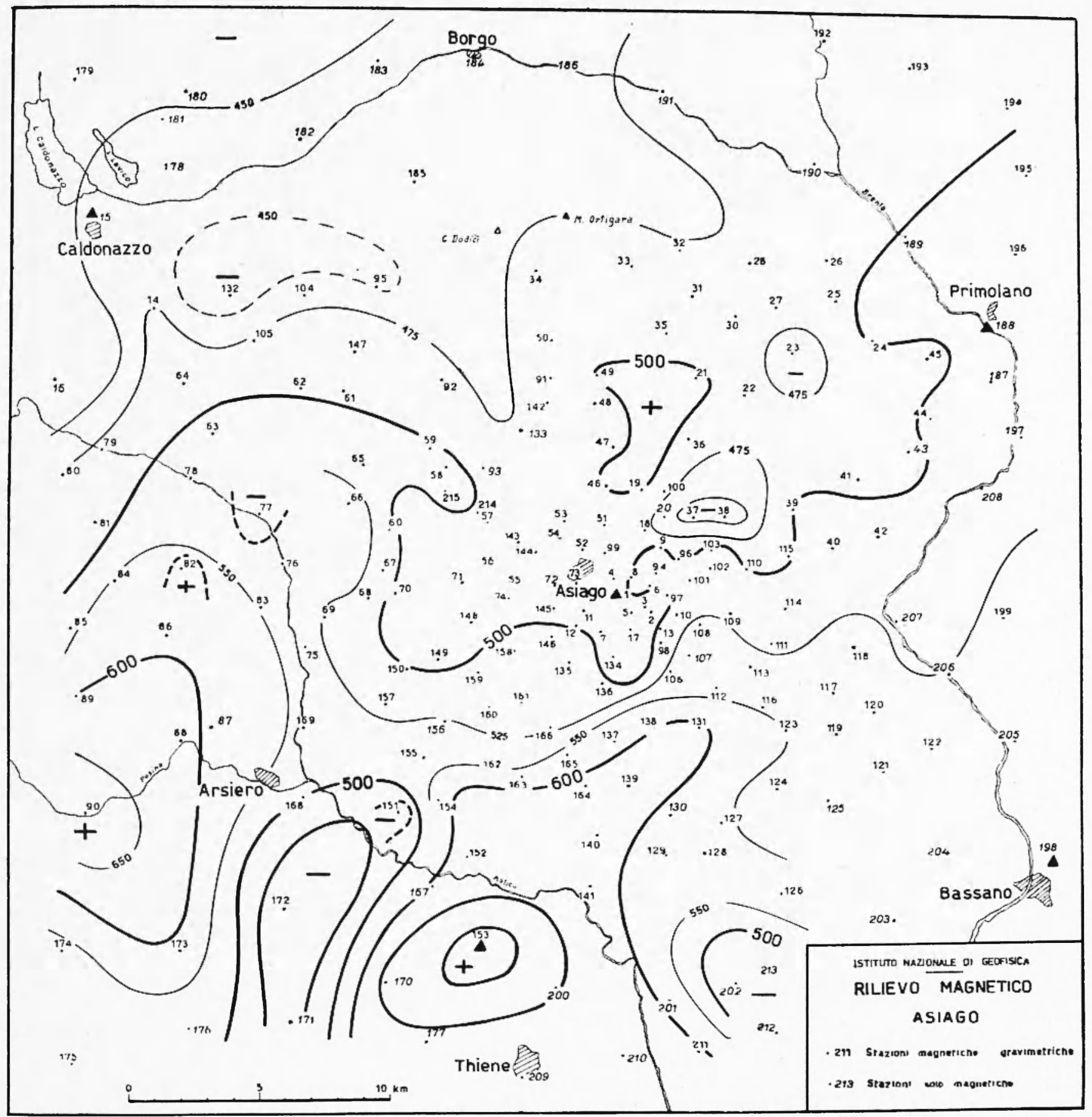

ISOANOMALE $(40.000 \gamma+\ldots)$ DELLA COMPONENTE VERTICALE DEL CAMPO MAGNETICO TERRESTRE

Fig. 1 
errori di chiusura di questi l'enore medio di una singola stazione risulta:

$$
m= \pm 4.8 \gamma
$$

Complessivamente, sono state eseguite 215 stazioni magnetiche, la cui distribuzione risulta dalla fig. 1 .

Nella stessa fig. 1 è riportata anche la distribuzione delle anomalie magnetiche, quali risultano dopo aver applicato ai valori, già ridolti per la variazione diurna e compensati per gli errori di chiusura dei circuiti, le correzioni normali di latitudine e di longitudine.

Essendo esse per la regione in esame ( ${ }^{1}$ )

$$
-6.065 \cdot \gamma / \mathrm{km} \quad \text { e } \quad+0.35 \gamma / \mathrm{km}
$$

sono state applicate in funzione delle differenze di latitudine e di longitudine di tutte le stazioni dal n. 1 , con i seguenti coefficienti:

$$
-0.19643 \gamma / 1^{\prime \prime} \quad \text { e } \quad+0.01621 \quad \gamma / 1^{\prime \prime} \text {. }
$$

3. Discussione delle anomalie magnetiche - Essendo la susceltività magnetica del calcare molto bassa, come del resto quella delle rocce sedimentarie ed eruttive acide (suscettività inferiore a $700.10^{6}$ ), mentre invece sono cospicui i valori connessi con le rocce cristalline ed eruttive basiche (fino a $15.000 .10^{\circ}$ ), la salita di queste da profondità tenderà orunque ad aumentare il valore delle anomalie osservate. Sicché in definitiva sarà lecito, almeno in generale, considerare quali valori magnetici normali per la zona in esame i più hassi, e riferire le differenze da questi al contributo delle masse vulcaniche soprammenzionate.

Nella zona dell'Altipiano di Asiago il massimo spessore di calcare, o almeno di calcare senza batoliti, si arreble quindi proprio nella zona della città di Asiago, con una radice locale più profonda verso NE.

La risalita del fondo sottostante alla dolomia sarebbe quindi più rapida al Sud di Asiago, mentre all'esterno dell'Altipiano, sul fronte meridionale, dominano le caratteristiche anomalie locali connesse con i basalti e le porfiriti. 
Domina ancora a SW la grande anomalia positiva di Posina, molto allungata nel senso della latitudine: essa è causata dalla massa porfritica intrusiva (laccolitica) M. Alba-Posina, e conferma così pienamente le intuizioni del prof. Fabiani (v. fig. 2).

Verso la parte Nord dell'Altipiano le anomalie magnetiche sono molto più regolari, segno questo che mancano in profondità le intrusioni batolitiche; e diminuiscono, in conseguenza probabilmente dell'aumentato spessore del calcare con l'aumentare della quota.

Anche nella zona della Val Sugana esaminata, e verso i laghi di Caldonazzo e Levico, i valori magnetici osservati sono regolari, in

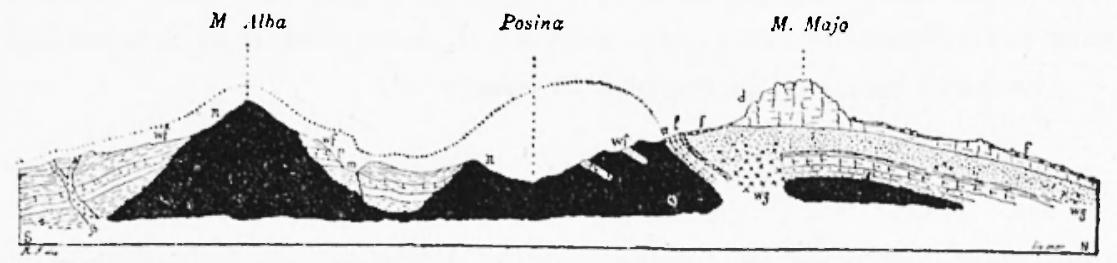

Fig. 2 - Sezione attraverso la massa porfiritica intrusiva (laccolitica) M. Alba-Posina, secondo Fabiani ( 2 ).

$m$, Micascisti; $p$, Permiano; $w f$, Werfeniano; $a$, Anisico; $\pi$, porfirite intrusiva; $l$, Ladinico inferiore (Bucbenstein); $w g$, formazioni eruttive e piroclastiche del Ladinico superiore (Wengen); $d$. Dolomia principale; $\beta$, filone di roccia basaltica; f, materiali di frani.

accordo con i bassi valori della suscettività magnetica delle rocce ivi esistenti.

In conclusione, possiamo dire che le misure magnetiche confermano l'omogeneità e la normalità delle condizioni sull'altipiano dei Sette Comuni, e precisano a Sud i limiti di penetrazione delle manifestazioni vulcaniche.

4. Rilievo gravimetrico. - Contemporaneamente al rilievo magnetico, è stato eseguito anche un rilievo gravimetrico. La difficoltà maggiore per una uniforme distribuzione delle stazioni gravimetriche è in questi casi, com'è noto, la necessità di eseguire le misure gravimetriche in punti di cui sia nota la quota: nella zona in esame ci si è riferiti, per quanto possibile, ai trigonometrici dell'Istituto Geografico Militare; e, dove questi mancavano, ai punti quotati delle tavolette al 25.000. Pochissime le possibilita di riferirsi ai caposaldi altimetrici della livellazione geometrica dell'I.G.M., oppure alla livellazione del piano del ferro delle FF.SS.

Lo strumento impiegato è stato il gravimetro Worden n. 50, con 


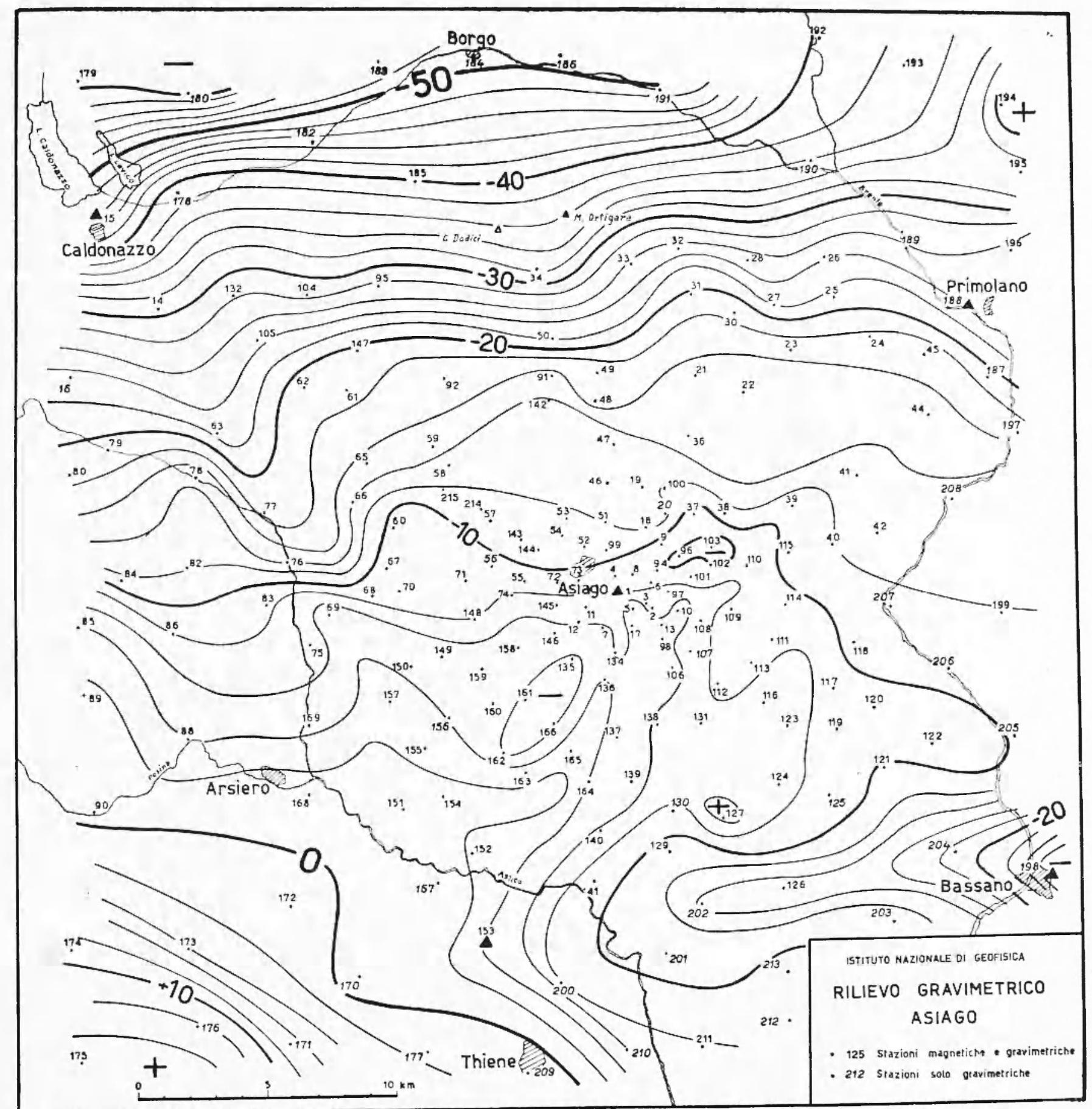

ISOANOMALE SECONDO BOUGUER - EQUIDISTANZA $2 \mathrm{mgal}$

Fig. 3 
i consueti criteri operativi e di riduzione ( $\left.{ }^{3}\right)$. Complessivamente sono state eseguile 206 stazioni gravimetriche, la cui distribuzione risulta dalla fig. 3 .

Lo schema delle densità adottate per il calcolo della calotta e della correzione topografica, estesa fino alla zona $L$, risulta dalla fig. 4 .

Nella fig. 5 sono riportati invece il campo regionale e le anomalie locali.

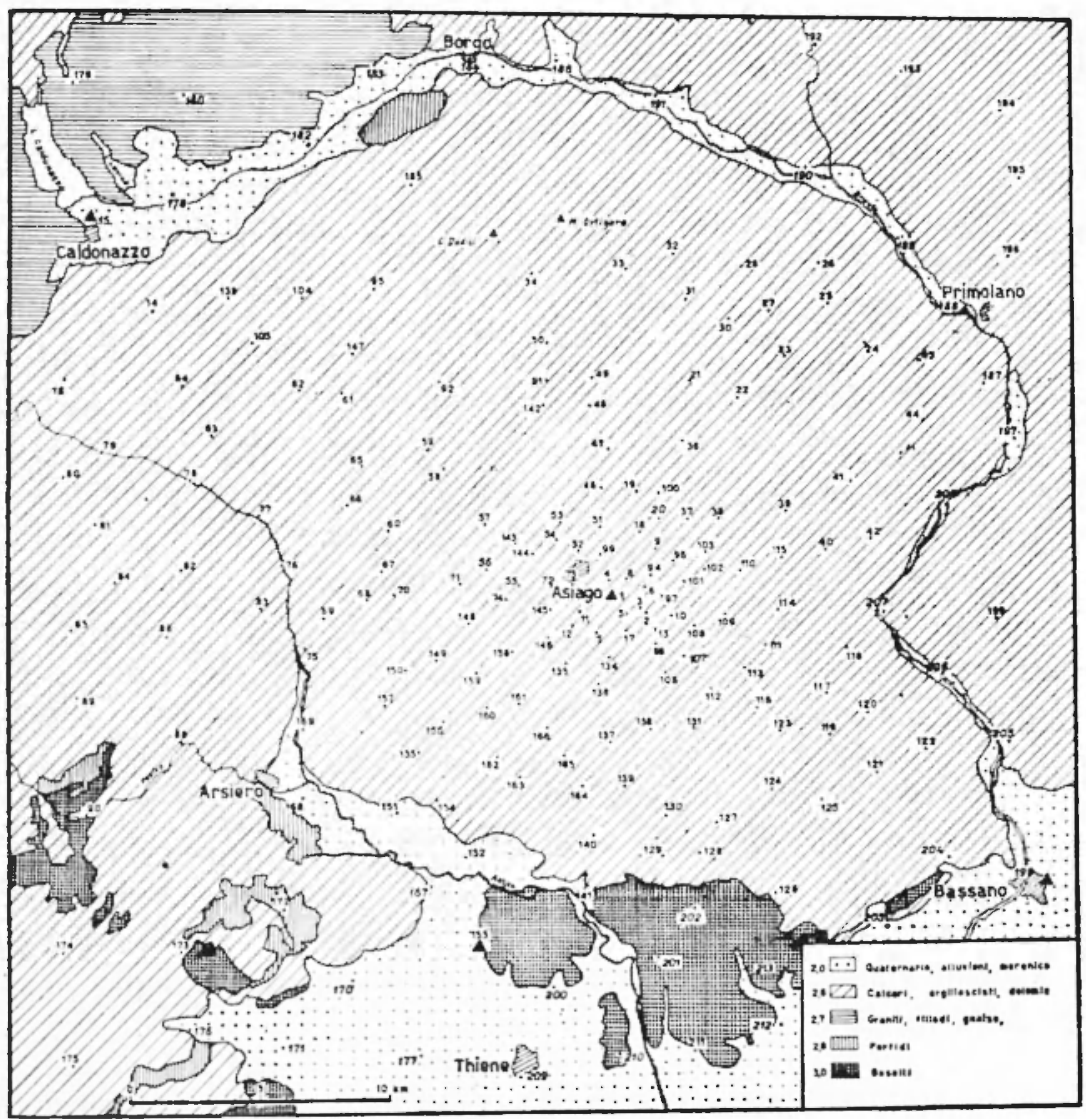

Fig. + - Differenziazione schematica delle densiti, per il calcolo della correzione per la calotta e topografiea.

Come stazione di riferimento si è fissato un punto davanti alla sogrlia della cupola dell'Osservatorio Astrofisico di Asiago, che è stato poi collegato, attraverso Carré, con la base di Vicenza ("). 
Il risultato ̀̀ stato il seguente:

\begin{tabular}{|c|c|c|}
\hline Stazione & $\begin{array}{l}\Delta \mathrm{g} \\
(m \boldsymbol{g} g l)\end{array}$ & $\stackrel{g}{g})$ \\
\hline $\begin{array}{l}\text { Vicenza } \\
\text { Carré } \\
\text { Asiago n. } 1\end{array}$ & $\begin{array}{r}-36.32 \\
-154.64\end{array}$ & $\begin{array}{r}980,681.60 \\
, 645.28 \\
, 490.64\end{array}$ \\
\hline
\end{tabular}

La monografia delle predette stazioni è la seguente:

Carré: Sotto il portico del fabbricato di fronte alla fermata ferroviaria $(\mathrm{km} 5+960)$.

Asiago: Osservatorio Astrofisico, a m 1.30 dalla soglia d'ingresso Sud alla Cupola.

5. Discussione delle anomalie gravimetriche. — L'esame della fig. 3 mostra anzitutto chiaramente come l'Altipiano di Asiago si trovi ( $\left.{ }^{3}\right)$ nella zona di transizione fra la grande anomalia gravimetrica negativa del centro delle $\mathrm{Al}_{\mathrm{p}} \mathrm{i}$, conseguente ai fenomeni responsabili dell'equilibrazione isostatica, e la grande anomalia positiva a Sud, di origine magmatica, le cui manifestazioni più cospicue esterne sono $i$ Berici - Lessini - Euganei.

In tale zona di transizione il campo gravimetrico risulta molto perturbato, per tre differenti motivi:

$1^{\circ}$ Carsismo. Nel calcare tale fenomeno è molto diffuso, per cui in tutto l'altipiano di Asiago si hanno dei grandi deficit di densità derivanti dalle cavità naturali, che in gran numero esistono nella zona (solo in minima parte note). Da ciò principalmente il gran numero di anomalie gravimetriche negative, quali risultano sia dalla fig. 3 che dalla fig. 5 .

$2^{\circ}$ Intrusioni magnatiche. In conseguenza della diminuzione dello spessore del Sial per effetto delle correnti di convezione magmatiche (teoria dell'Einchnickung (") di Vening Meinesz), tutta la zona è caratterizzata da intrusioni magmatiche molto diffuse, sia a Sud dell'altipiano calcareo che al di sotto del suo bordo meridionale. Cró risulta chiaramente sia dalle anomalie gravimetriche che da quelle magnetiche, e conferma le intuizioni dei geologi $\left(^{2}\right)\left({ }^{6}\right)$. 


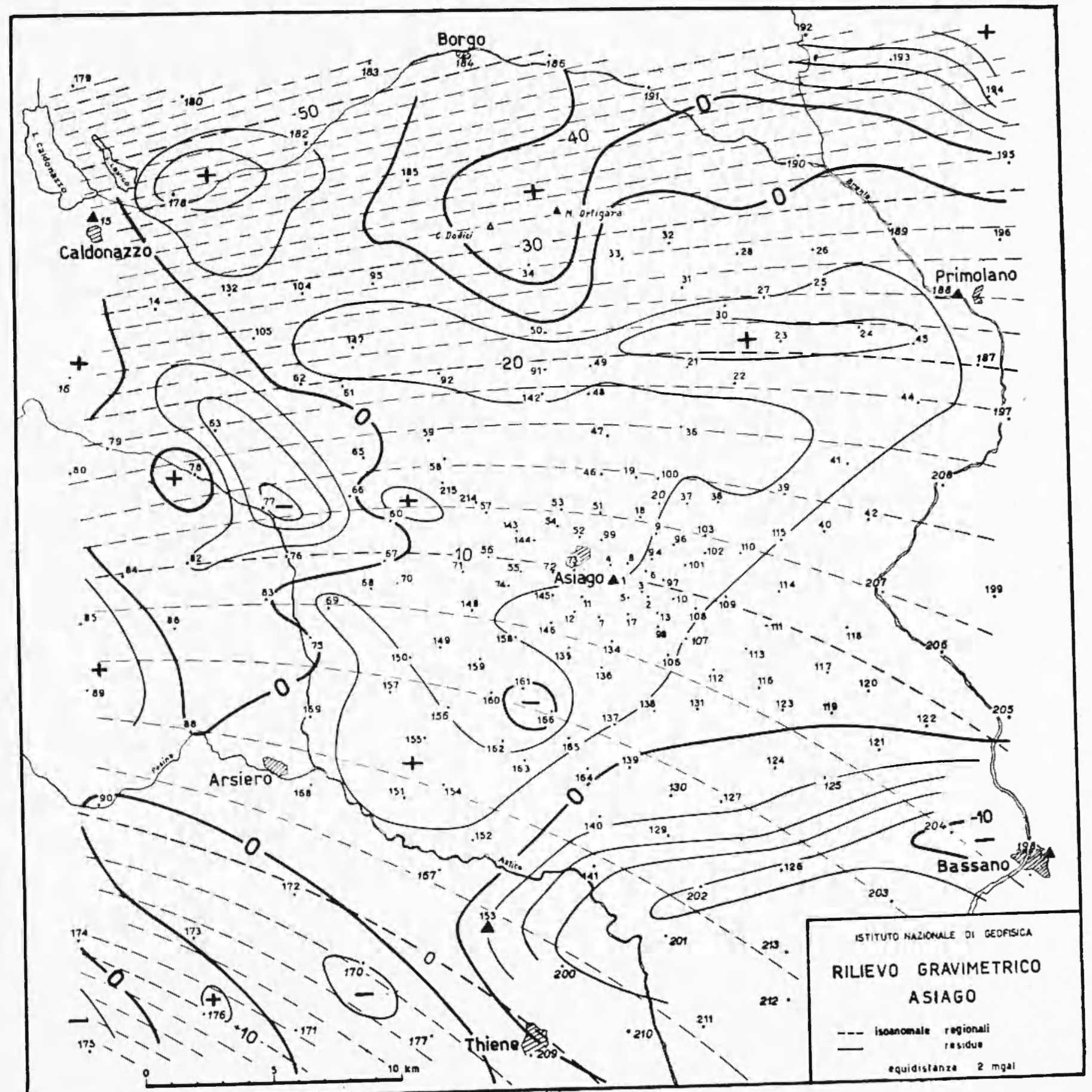

Fig. 5 - Campo gravimetrico ed anomalie locali. 
$3^{\circ}$ Pieghe, prevalentemente a ginocchio, al bordo merid. Tale tipo di pieghe $\left({ }^{2}\right)\left({ }^{\top}\right)$, comportando il trascinamento verso il basso di materiale meno denso ( $f g$. 6), ¿̀ ugualmente connesso con anomalie

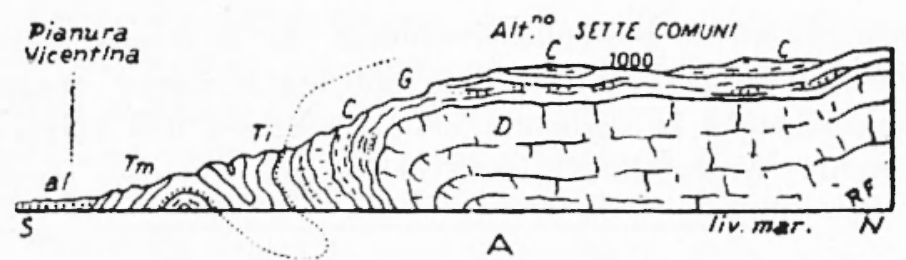

Fig. 6 - Piega a ginorchio al marrine Sud dell'Altipiano di Asiago, secondo Fabiani $(\sigma)$.

$D$, Dolomia del Trias sup.; $G$, Giurese; $C$, Cretaceo; $T$, Terziario inf.; Tm, Terziario medio; Al, Alluvioni.

gravimetriche negative: tale causa è quindi da attribuirsi probabilmente alla grande anomalia gravimetrica negativa sul margine Sud dell'Alipiano.

Oppure si può ammettere che questa sia l'estrema propaggine nord-occidentale della grande sinclinale di fondo a SE di Bassano $\left({ }^{3}\right)$,

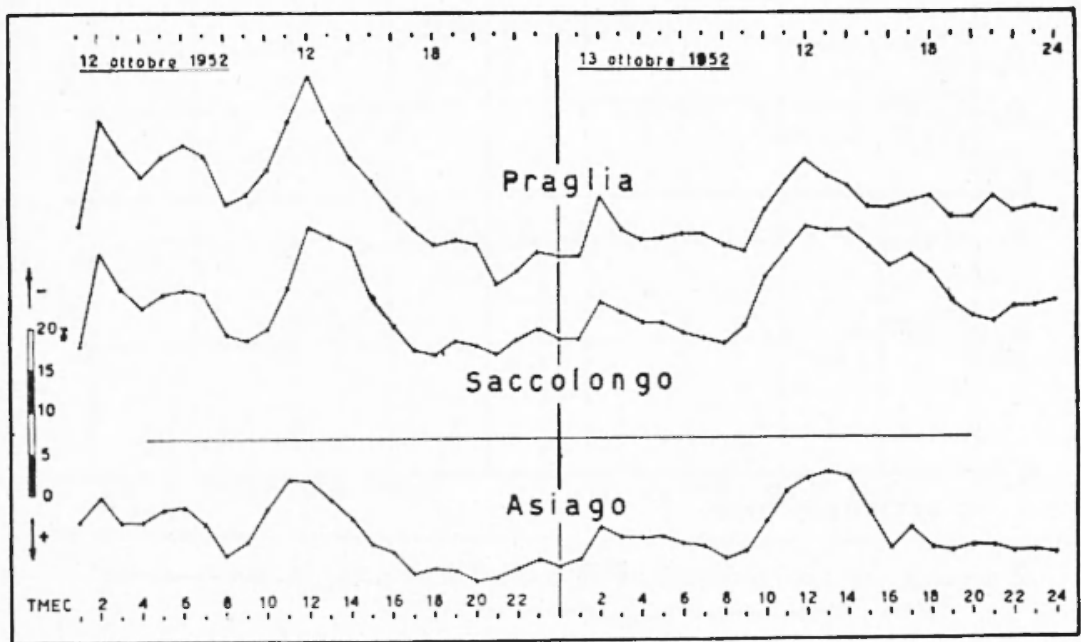

Fig. 7 . Variazione diuma della $Z$ in tre differenti stazioni (Praglia = Euganei; Sacrolongo $=$ avampaese degli stessi; Asiago).

con consequente trascinamento verso il basso delle pieghe sopraddette.

L'esame del campo regionale ( $f i g .5$ ) conferma il carattere di zona di transizione sopra menzionato, fra il gradiente negativo molto forte a Nord e il gradiente positivo altrettanto forte a Sud. 
Le anomalie residue, oltre a quella negativa molto spiccata a SE di cui si è già parlato, ne registrano un'altra negativa bene individuata anche al margine $\mathbb{W}$ dell'Altipiano, probabilmente doruta a motivo analogo.

Anche da queste possiamo concludere che le misure gravimetriche confermano quanto già avevano indicato le misure magnetiche, cioè l'omogeneità e la normalità delle condizioni dell'Altipiano dei Sette Comuni; e precisano i limiti dell'attivita teltonica circostante.

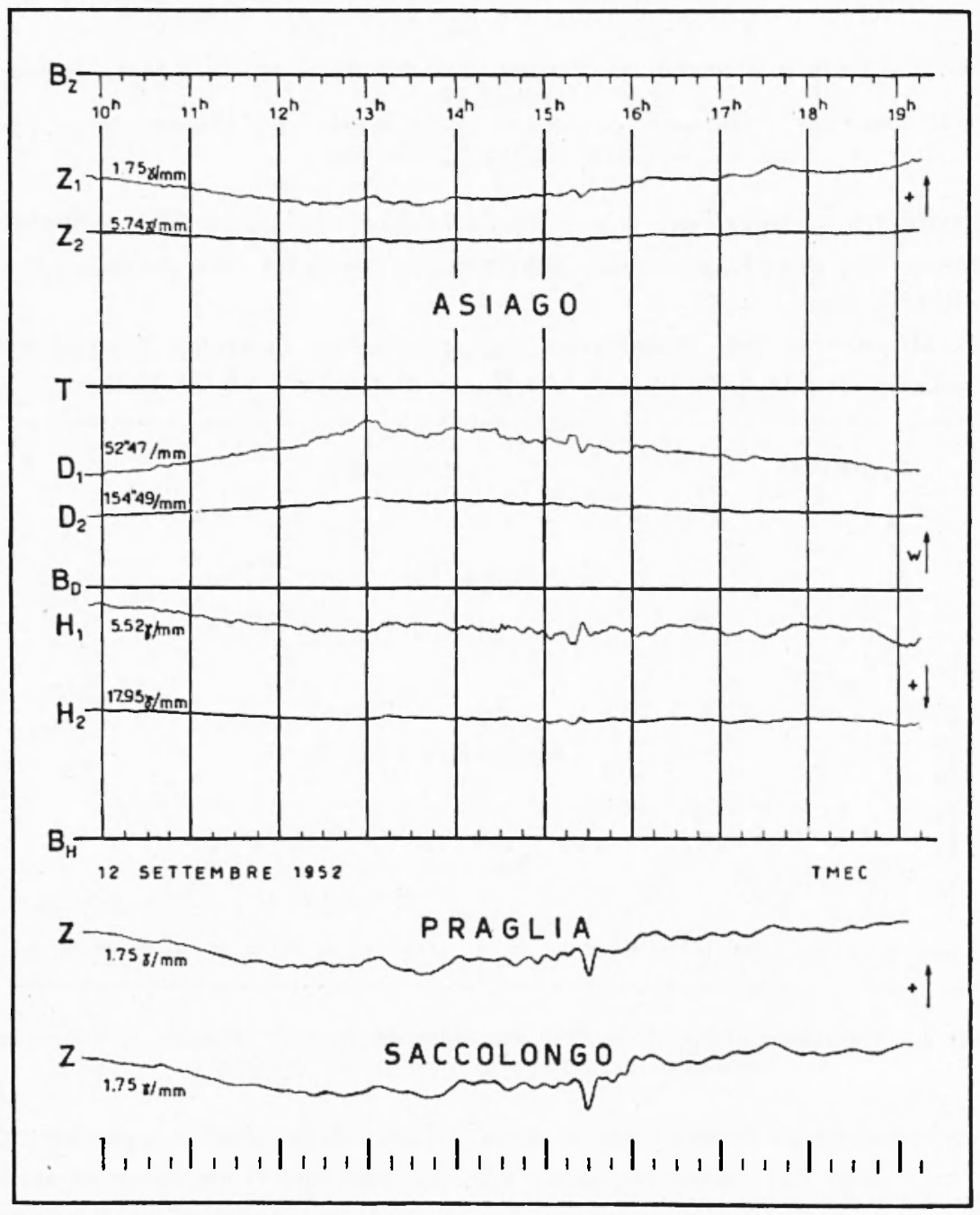

Fig. 8 - Esempio di diagramma completo per Asiago, con micropulsazioni anche a Praglia e Saccolongo. 
In generale poi, anomalie gravimetriche e magnetiche presentano un quadro notevolmente diverso, come appunto dev'essere, derivando da fenomeni fisici differenti (se pure per altri aspetti connessi).

6. Variazioni magnetiche nel tempo. - Oltre che alle variazioni geofisiche nello spazio, era importante studiare il comportamento dell'Altipiano di Asiago alle variazioni magnetiche nel tempo. Un anno di registrazioni della stazione sopra menzionata a tre componenti, in funzione provvisoriamente presso l'Osservatorio Astrofisico di Asiago, hanno dimostrato ormai la assoluta normalita delle condizioni ma-

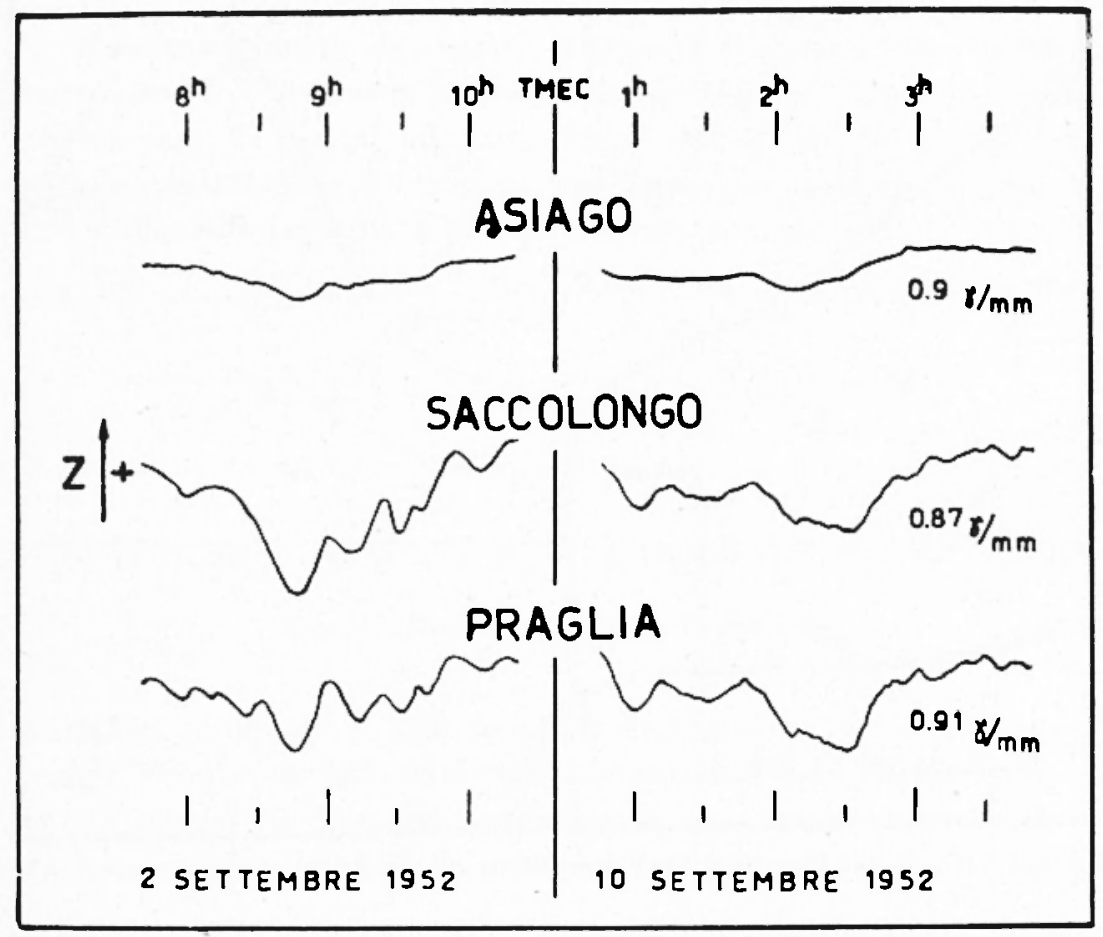

Fig. 9 - Casi di micropulsazioni sugli Euganei, e non ad Asiago.

gnetiche sull'Altipiano. Nessuna causa di perturbazione è presente, né è apprezzabile l'influsso di eventuali cause lontane.

Una prova diretta si è potuta avere anche dal confronto fra le registrazioni di Asiago e quelle di due stazioni registratrici provvisorie, allestite a Praglia $\left(45^{\circ} 21^{\prime}, 7 \mathrm{~N}, 0^{\circ} 43^{\prime}, 0 \mathrm{~W}\right.$ Monte Mario: sugli Euganei) e Saccolongo $\left(45^{\circ} 24^{\prime}, 3 \mathrm{~N}, 0^{\circ} 42^{\prime}, 1 \mathrm{~W}\right.$ : a Nord degli Euganei) in occasione del rilievo magnetico e gravimetrico dell'avampaese degli 
stessi. Queste due stazioni erano costituite da due bilancie magnetiche Askania, con testa di registrazione a cellula fotoelettrica e registratore a galvanometro; sensibilità :

Praglia $0,9097 \gamma / \mathrm{mm} \quad ;$ Saccolongo $0,3721 \gamma / \mathrm{mm}$.

Hanno funzionato, se pur non continuamente, dal 2 settembre al 19 novembre 1952.

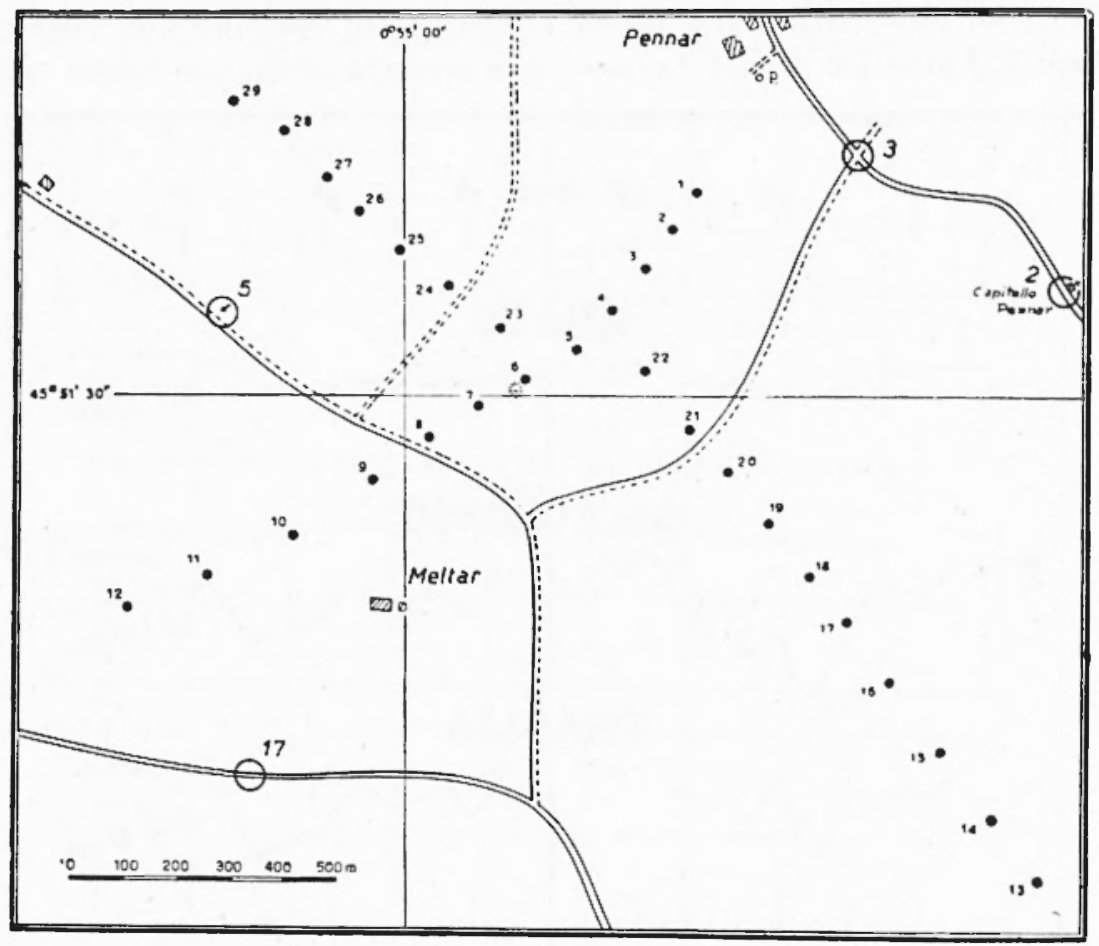

Fig. 10 - Zona prescelta a SE di Asiago.

La similitudine della variazione diurna registrata nelle tre località è stata sempre perfetta; solo l'ampiezza sugli Euganei risulta un po' esaltata.

Riportiamo in fig. 7 un esempio di tale variazione.

Anche le microvariazioni del campo magnetico terrestre si ritrovano fedelmente riprodotte ad Asiago, come del resto nelle altre due stazioni. Riportiamo un esempio in fig. 8. Anche ora, l'ampiezza sugli Euganei è maggiore: interessante fenomeno, da meritare una ricerca a parte. Tanto più che in qualche (raro) caso, sono state registrate 
micropulsazioni identiche a Praglia e Saccolongo, e nessuna traccia ad Asiago (fig. 9).

Ad ogni modo, quello che interessava mettere in evidenza era la normalità di Asiago rispetto alle variazioni magnetiche: e questa è slata provata anche dai confronti qui menzionati.

7. Conclusioni. - In conclusione, possiamo dire che l'Altipiano di Asiago si presta per la costruzione di un Osservatorio magnetico di $1^{\circ}$ ordine come ben poche altre località nell'Italia sett.

Rimaneva ancora da cercare il posto più idoneo per l'eventuale costruzione dell'Osservatorio stesso. Considerazioni di natura logistica consigrliavano di non allontanarsi troppo dall'abitato di Asiago e dall'attuale Osservatorio Astrofisico; una zona pianeggiante, aperta, con tutti i requisiti richiesti venne fortunatamente trovata a $3 \mathrm{~km}$ a $\mathrm{SE}$

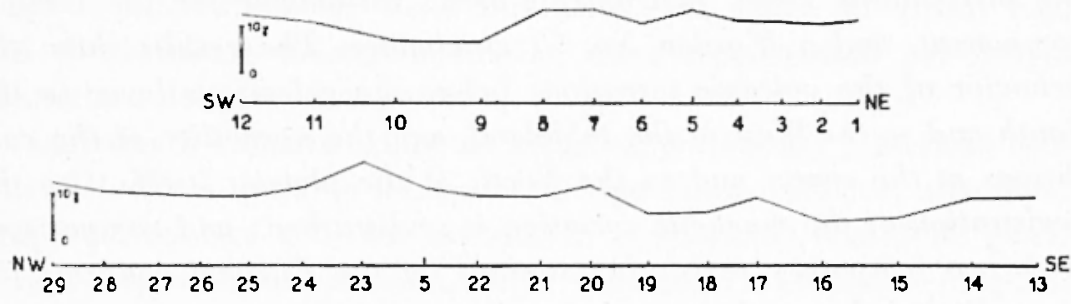

Fig. 11 - Risultati del rilievo di dettaglio.

di Asiagro, e quindi a $1250 \mathrm{~m}$ a SE dell'Osservatorio Astrofisico, in localita Pennar (a Sud delle stazioni magnetiche 2, 3 e 5; v. fig. 1).

Un rilievo di dettaglio, costituito da una sezione trasversale ed una longitudinale ( fig. 10), ha dato risultati del tutto normali (fig. 11). Istituto Nazionale di Geofisica - Osserv. di Trieste - Febbraio 1953.

\section{RIASSUNTO}

Per studiare il comportamento geofisico dell'Altipiano calcareo di Asiago, è stato eseguito nell'agosto 1952 un rilievo magnetico di 215 stazioni ed un rilievo gravimetrico di 206 stazioni, sull'Altipiano stesso e zone circostanti. Strumenti impiegati: variometro per la componente 
verticale, e gravimetro Worden n. 50. I risultati precisano l'andamento delle intrusioni vulcaniche al di sotto del calcare, a Sud e ad Ovest dell'Altipiano; e la normalità delle condizioni al centro ed al Nord dell'Altipiano stesso. Anche la registrazione delle variazioni magnetiche risulta indisturbata; $e$ il confronto con due stazioni registratrici temporanee sugli Euganei conferma la normalità delle condizioni sull'Altipiano di Asiago.

\section{$S U M M A R Y$}

In order to study the geophysical behavior of the calcarious plateau of Asiago, in August 1952 a magnetic relief of 215 stations and a gravimetric relief of 206 stations were placed on the plateau itself and the surrounding zones. Instruments used: variometer for the vertical component, and a Worden No. 50 gravimeter. The results show the behavior of the volcanic intrusions below the calcarious layer to the South and to the West of the tableland, and the normality of the conditions at the center and to the North of the plateau itself. Also the registration of the magnetic variation is undisturbed; and comparisons with two temporary registering stations on the Euganei confirms the normality of the conditions on the plateau of Asiago.

\section{BIBLIOGRAFIA}

(1) Monelli C.: Teoria e pratica dei variometri magnetici da campagna. Ed. Del Bianco, 164 pg., Udine 1947.

(2) Fabiani R.: La regione del Pasubio. Uff. Idrografico del Magistrato delle Acque, pubbl. n. 110, Venezia 1920.

(3) Morelli C.: Rilievo gravimetrico e riduzioné isostatica nell'talia nordorientale. Tecnica Italiana, Nuova Serie, VI, 3 e 4, Trieste 1951.

(4) Monelli C.: Primo contributo per una rete gravimetrica fondamentale in Italia. Annali di Geofisica, V, 1, Roma 1952.

(5) Veccha O.: Sui principali caratteri strutturali dellItalia sett. dedotti dalle misure gravimetriche. Riv. di Geofisica appl., XIII, 1, 33-67, Milano 1952.

${ }^{(6)}$ Fabiani R.: Trattato di Geologia. Istituto Grafico Tiberino, Roma 1952.

(7) Dal PIaz G.: Studi geotetlonici sulle Alpi orientali. Mem. Ist. Geol. Univ. Padova, vol. I (1908-12). 